\title{
Evaluations of Mo-alloy for light water reactor fuel cladding to enhance accident tolerance
}

\author{
Bo Cheng ${ }^{1 *}$, Peter $\mathrm{Chou}^{1}$, and Young-Jin Kim ${ }^{2}$ \\ ${ }^{1}$ Electric Power Research Institute (EPRI), Palo Alto, CA 94304, USA \\ ${ }^{2}$ GE Global Research Center, Schenectady, NY 12309, USA
}

Received: 21 September 2015 / Received in final form: 26 November 2015 / Accepted: 3 December 2015 Published online: 1 February 2016

\begin{abstract}
Molybdenum based alloy is selected as a candidate to enhance tolerance of fuel to severe loss of coolant accidents due to its high melting temperature of $\sim 2600{ }^{\circ} \mathrm{C}$ and ability to maintain sufficient mechanical strength at temperatures exceeding $1200^{\circ} \mathrm{C}$. An outer layer of either a $\mathrm{Zr}$-alloy or Al-containing stainless steel is designed to provide corrosion resistance under normal operation and oxidation resistance in steam exceeding $1000^{\circ} \mathrm{C}$ for 24 hours under severe loss of coolant accidents. Due to its higher neutron absorption cross-sections, the Mo-alloy cladding is designed to be less than half the thickness of the current Zr-alloy cladding. A feasibility study has been undertaken to demonstrate (1) fabricability of long, thin wall Mo-alloy tubes, (2) formability of a protective outer coating, (3) weldability of Mo tube to endcaps, (4) corrosion resistance in autoclaves with simulated LWR coolant, (5) oxidation resistance to steam at $1000-1500{ }^{\circ} \mathrm{C}$, and (6) sufficient axial and diametral strength and ductility. High purity Mo as well as $\mathrm{Mo}+\mathrm{La}_{2} \mathrm{O}_{3} \mathrm{ODS}$ alloy have been successfully fabricated into $\sim 2$-meter long tubes for the feasibility study. Preliminary results are encouraging, and hence rodlets with Mo-alloy cladding containing fuel pellets have been under preparation for irradiation at the Advanced Test Reactor (ATR) in Idaho National Laboratory. Additional efforts are underway to enhance the Mo cladding mechanical properties via process optimization. Oxidation tests to temperatures up to $1500^{\circ} \mathrm{C}$, and burst and creep tests up to $1000{ }^{\circ} \mathrm{C}$ are also underway. In addition, some Mo disks in close contact with $\mathrm{UO}_{2}$ from a previous irradiation program (to $>100 \mathrm{GWd} / \mathrm{MTU}$ ) at the Halden Reactor have been subjected to post-irradiation examination to evaluate the chemical compatibility of Mo with irradiated $\mathrm{UO}_{2}$ and fission products. This paper will provide an update on results from the feasibility study and discuss the attributes of the coated Mo cladding design to meet the challenging requirements for improving fuel tolerance to severe loss of coolant accidents.
\end{abstract}

\section{Introduction}

Zr-based alloys have served as the fuel cladding for light water reactors due to unique combination of low neutron cross-section, adequate corrosion resistance and mechanical properties. The reliability of Zr-alloy cladding has been steadily improved over the last five decades and has reached an excellent status in recent years. The Fukushima Daiichi accident triggered by the tsunami following an earthquake has illustrated the vulnerability of Zr-alloys to rapid steam oxidation during a severe accident when the flow of coolant into the reactor core is interrupted. Without availability of coolant flow to remove the nuclear decay heat, the fuel cladding temperature will rise rapidly. At temperatures exceeding $700-1000{ }^{\circ} \mathrm{C}$, depending on the steam pressure, exothermic reaction of $\mathrm{Zr}$ with steam will release hydrogen and enthalpy or heat when $\mathrm{ZrO}_{2}$ is formed. Due to the high

\footnotetext{
* e-mail: bcheng@epri.com
}

packing density of fuel rods in reactor cores, where a typical size core may have $\sim 50,000$ fuel rods, the total heat from zirconium oxidation may exceed that of the nuclear decay heat, if oxidation heat is all released within a short duration of an hour or so. The excessive oxidation heat may contribute to earlier melting of some reactor core components and subsequently the fuel pellets. The amount of hydrogen generated by zirconium oxidation can be in the order of exceeding $1,000 \mathrm{~kg}$, and, hence, can complicate efforts by plant operators to recover the cooling system to stabilize the plant [1].

For current LWRs, it is most essential to maintain availability of coolant flow into the core under any accident conditions, and the FLEX program initiated by the US NRC is targeted to achieving that objective. Another potential defense is to replace the zirconium alloy fuel cladding with another material having substantially higher resistance to steam oxidation and capability of maintaining fuel rod integrity at elevated temperatures, which may provide meaningful coping time for plant operators to 


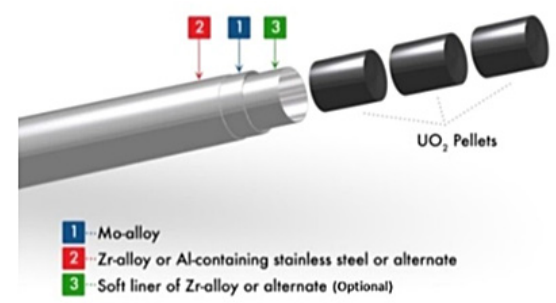

Fig. 1. Schematic of coated Mo-alloy cladding.

restore the core cooling systems [2,3]. The US Department of Energy (DOE) launched a multi-year R\&D program with funding to the national laboratories and nuclear fuel vendors to develop enhanced accident tolerant fuel (ATF) in 2012 [2]. Various international programs have also been launched over the last 3 years [4]. Candidate new cladding materials have included: coated $\mathrm{Zr}$-alloy, $\mathrm{SiC}_{-} \mathrm{SiC}_{\mathrm{f}}$ composite, Al-containing stainless steel and refractory metal (primarily molybdenum alloy).

EPRI initiated conceptual designs of coated molybdenum alloy cladding by utilizing the high temperature strength $\left(1500{ }^{\circ} \mathrm{C}\right.$ and beyond) of molybdenum to maintain core geometry for coolability during a design based and beyond the design based loss of coolant accidents, as illustrated in Figure $1[1,3]$. Metallurgically bonded surface coating with Al-containing stainless steel or Zr-alloy is to provide corrosion resistance during normal operation and steam oxidation resistance during loss of coolant accident with a target of surviving in steam at $1200-1500^{\circ} \mathrm{C}$ for at least 24 hours. The inner surface may have a soft liner layer as an option, but its need will be determined following completion of the feasibility study.

This paper outlines the scope of the feasibility study for coated Mo-alloy cladding and shares the results obtained to date, as well as discusses the challenges ahead for completion of the feasibility study.

\section{Scope of feasibility study and results}

The feasibility study focuses on the following topics:

- fabricate Mo-alloy tubes with $0.2-0.25 \mathrm{~mm}$ wall thickness and characterize mechanical properties;

- form metallurgically bonded coating with Al-containing stainless steel or Zr-alloy of $\sim 0.05 \mathrm{~mm}$;

- weld Mo-alloy tube to endcaps;

- characterize corrosion resistance of coated and uncoated Mo-alloy tubes in autoclaves with simulated LWR coolants;

- characterize steam oxidation of coated and uncoated Moalloy tubes in steam at $1000-1500{ }^{\circ} \mathrm{C}$.

Rodlets with coated Mo-alloy cladding containing enriched fuel pellets are also being fabricated for irradiation at the ATR reactor under funding by the US DOE and the Halden Reactor under its base program funding. Postirradiation characterization of high purity Mo disks and cladding rings which were previously irradiated to high burnup at the Halden Reactor is also included in this feasibility study.
The effects of higher neutronic absorption cross-sections of Mo on fuel economics and fuel cycle designs were assessed and reported previously [1].

\subsection{Fabrication of thin wall Mo-alloy tubes and mechanical properties}

Mo-based alloys have been used in reducing environments as containers or thermocouples at elevated high temperatures as high as $\sim 2000^{\circ} \mathrm{C}$. An extensive test program was undertaken by Bettis and Oak Ridge National Laboratories to evaluate the irradiation properties of various Mo-alloys in coupon forms for potential space reactor fuel material applications $[5,6]$. Thin wall Mo-alloy cladding suitable for LWR applications was not previously available. Under this ATF program, thin wall Mo-alloy tubes with the outer diameter of 9.4 or $10 \mathrm{~mm}$ ( 0.37 or 0.40 inches) and wall thickness of $0.2-0.25 \mathrm{~mm}$ have been fabricated in length of 1.5 meters $(5 \mathrm{ft})$. Tubes have been made of pure Mo, including from low carbon arc cast (LCAC) and powder metallurgy (PM) billets, as well as oxide dispersion strengthened Mo-alloy (Mo-DOS) in which Mo is doped with dispersed $\mathrm{La}_{2} \mathrm{O}_{3}$. Typical $\mathrm{La}_{2} \mathrm{O}_{3}$ concentration in weight is $\sim 0.3 \%$, but some tubes with $1 \%$ were fabricated. Both LCAC Mo and Mo-ODS were found to retain small residual ductility following irradiation performed by the Bettis/Oak Ridge Program. Excellent tube straightness and uniform wall thickness have been achieved (Fig. 2).

Because of its high melting temperature and single phase structure (i.e., absence of phase transition) until melting at $\sim 2600^{\circ} \mathrm{C}$, the mechanical strength will be maintained to much higher temperatures than that of common structural alloys including $\mathrm{Zr}, \mathrm{Fe}$ and $\mathrm{Ni}$ based alloys. Pure Mo can maintain tensile strength of $\sim 69 \mathrm{MPa}$ ( $\sim 10 \mathrm{ksi})$ at $1500^{\circ} \mathrm{C}$, while most other alloys would have lost their strength below $1000^{\circ} \mathrm{C}$.

The thin wall Mo and Mo-ODS tubes can achieve a tensile strength of $500-600 \mathrm{MPa}$ in partially recrystallized form with good axial fracture elongation of $20-25 \%$ when measured with $3.8 \mathrm{~cm}(1.5 ")$ gauge length tube at room temperature as shown in Table 1 . The strength can be controlled at $250-500 \mathrm{MPa}$ depending on the final annealing condition.

One common issue associated with high strength, thin wall tubes is their propensity to axial split. A test rig with internally pressurized argon gas has been developed to test the diametral properties, including the diametral failure strength and diametral strain and creep rate at temperatures

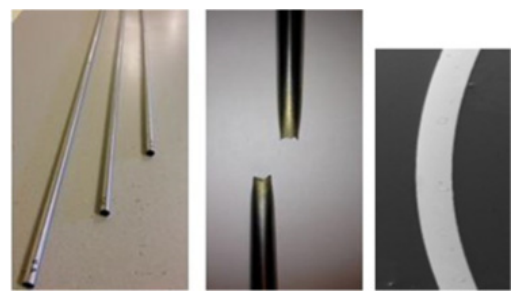

Fig. 2. Mo-alloy tubes with wall thickness of $0.2 \mathrm{~mm}$ and $\mathrm{OD}$ of $1.0 \mathrm{~cm}$. 
Table 1. Mechanical properties of partially recrystallized Mo-alloy tubes tested at room temperature.

\begin{tabular}{lccl}
\hline \multicolumn{4}{c}{ Tensile property (1.5” gauge length), $320^{\circ} \mathrm{C}$} \\
\hline & PM Mo partial recrystallization & \\
\hline & 1 & 2 & 3 \\
\hline $0.2 \%$ yield $(\mathrm{ksi}, \mathrm{MPa})$ & $44.5(307)$ & $50.6(349)$ & $53.7(370)$ \\
UTS (ksi, MPa) & $60.1(415)$ & $57.7(398)$ & $61(421)$ \\
Uniform elongation, $\%$ & 17 & 18 & 15 \\
Total elongation, $\%$ & 20 & 22 & 24 \\
\hline
\end{tabular}

up to $900^{\circ} \mathrm{C}$. Figure 3 illustrates the internally pressurized test results of samples of a partially recrystallized LCAC Mo tube. The measured diametral failure strength at $350{ }^{\circ} \mathrm{C}$ is $\sim 380 \mathrm{MPa}(55 \mathrm{ksi})$ and the diametral strain was measured as $1.4-16 \%$.

Optimizations of the mechanical properties, particularly the diametral properties, are in progress via two approaches: firstly, modification of the tube thermo-mechanical reduction process and, secondly, controlling the microstructure of the finished tubes. For microstructure control, an induction heat treatment chamber has been set up to heat treat tube samples to various temperatures in the $1000-1700^{\circ} \mathrm{C}$ range for $5-30$ seconds, in order to determine the optimum heat treatment temperature for achieving the desired diametral and axial mechanical properties. Preliminary data show that diametral strain of $6-18 \%$ can be achieved at lower diametral strength.

Attempts have also been made to control the microstructure with very fine grain size, which has been reported to increase both the strength and ductility of molybdenum [7]. Data to date has indicated that rapid heat treatment and cooling via induction heat treatment cannot accomplish grain size control for high purity Mo or Mo-ODS. Further evaluation with alloying of the Mo matrix has been planned.

\subsection{Formation of surface protective coating and interface stability}

Molybdenum is susceptible to accelerated corrosion and oxidation in oxidizing environments at $>\sim 300^{\circ} \mathrm{C}$ forming soluble and volatile $\mathrm{MoO}_{3}$. Due to the lack of technical basis for alloying Mo to improve its corrosion and oxidation resistance at present, surface protection via a corrosion resistant outer layer, as depicted in Figure 1, has been pursued for this ATF design. A limited effort of evaluating corrosion resistant Mo-alloys, such as Mo-Nb binary alloys, has been explored in parallel.

FeCrAl alloys with $\sim 20 \% \mathrm{Cr}$ and $\sim 6 \% \mathrm{Al}$ has been known to possess excellent corrosion resistance in simulated LWR coolants relying on the formation of a protective $\mathrm{Cr}_{2} \mathrm{O}_{3}$, as well as excellent oxidation resistance in steam to $\sim 1450{ }^{\circ} \mathrm{C}$ owing to the formation of a thin $\mathrm{Al}_{2} \mathrm{O}_{3}$ protective oxide. Zr-alloys can be optimized to possess excellent corrosion resistance in LWRs, and will convert to $\mathrm{ZrO}_{2}$ rapidly at $>1000^{\circ} \mathrm{C}$, which is expected to be stable in steam to protect the underlying Mo cladding.

This ATF project was designed to first prepare Mo-alloy tube samples with an outer protective layer utilizing suitable surface deposition techniques for various testing to

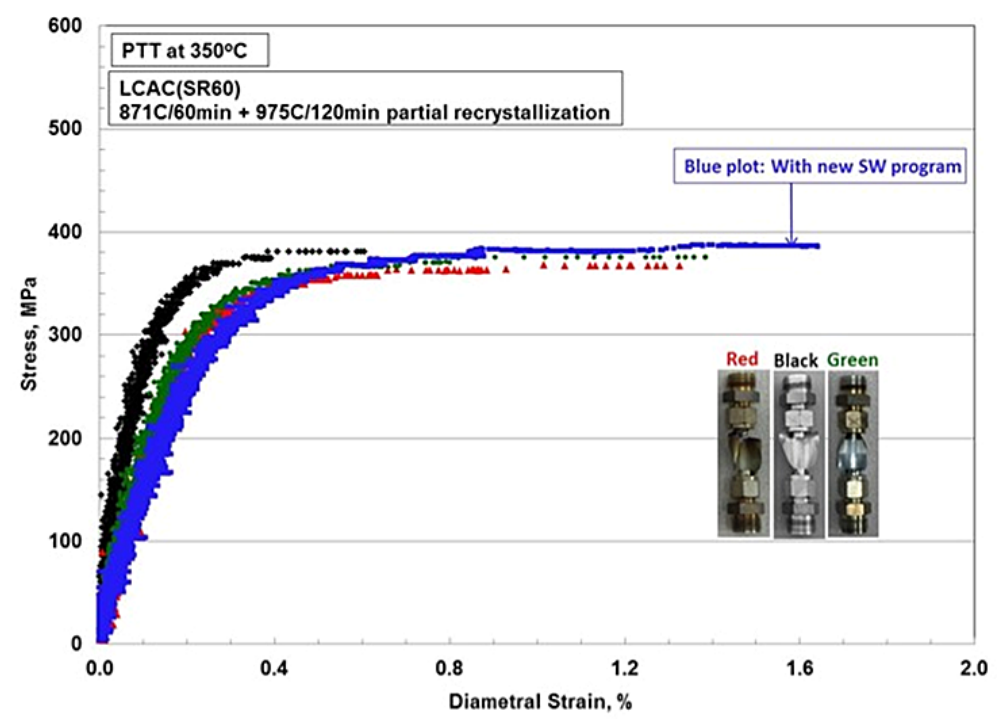

Fig. 3. Internally pressurized test of samples from a partially recrystallized LCAC Mo tube at $350{ }^{\circ} \mathrm{C}$. 


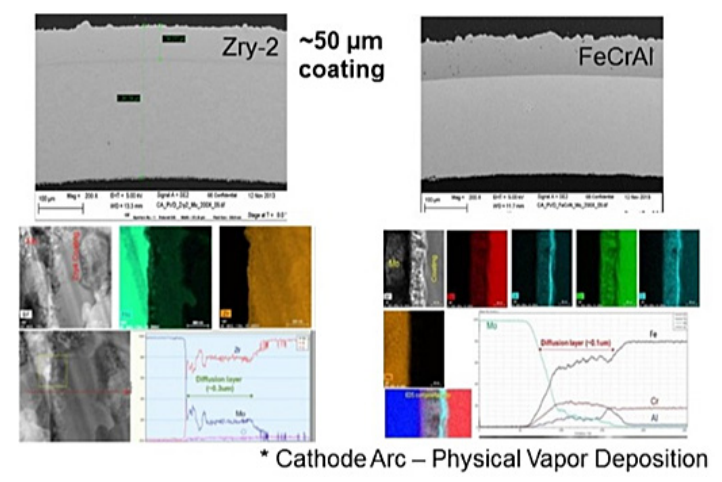

Fig. 4. Cross-section views of Mo tube with $0.2 \mathrm{~mm}$ wall thickness coated with a Zircaloy-2 or FeCrAl layer of $\sim 0.05 \mathrm{~mm}$. The pictures at the bottom show the presence of an inter-diffusion layer of 0.3 and $0.1 \mu \mathrm{m}$ at the interface of the Zircaloy- 2 and $\mathrm{FeCrAl}$ coating, respectively [10].

demonstrate feasibility of the ATF design. Tasks have been implemented to evaluate the feasibility of mechanical coreduction. The outer coating is required to form a metallurgical bonding with the Mo cladding in order to achieve integrity in all conditions.

Formation of a corrosion and oxidation resistant outer layer of Al-containing stainless steel or Zr-alloy have been successfully developed using various deposition techniques. Cathode Arc Physical Vapor Deposition, or CA-PVD, has been found to achieve the deposited layer with (1) excellent thickness uniformity, (2) excellent adhesion of the coating to the Mo tube, (3) excellent metal density with no visible porosity of the coating. Examples of CA-PVD coated Mo tubes are illustrated in Figure 4. It can be seen an interdiffusion layer of 0.3 and $0.1 \mu \mathrm{m}$ forms at the interface of the Zircaloy-2 and FeCrAl coating, respectively. The interdiffusion layer provides excellent metallurgical bonding of the coated layer to the Mo tube. Figure 5 shows a uniform coating of $\mathrm{FeCrAl}$ formed on a welded Mo tube.

\subsection{Tube to endcap welding}

Mo tube to endcap welding has been successfully demonstrated via plasma and tungsten inert gas welding as well as electron beam (EB) welding. EB welding has been found to produce smaller weld and heat affected zones, as illustrated in Figure 6a, and has been used to fabricate rodlets for irradiation at the Advanced Test Reactor (ATR) in Idaho National Laboratory (INL).

Resistance projection welding has been demonstrated to fuse Mo tube to endcap at the interface without forming a

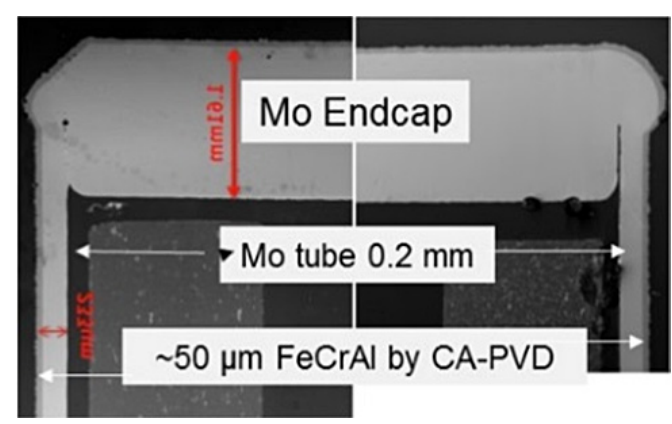

Fig. 5. Uniform FeCrAl coating thickness of $50 \mu \mathrm{m}$ on a welded Mo tube (similar results for Zircaloy coating).
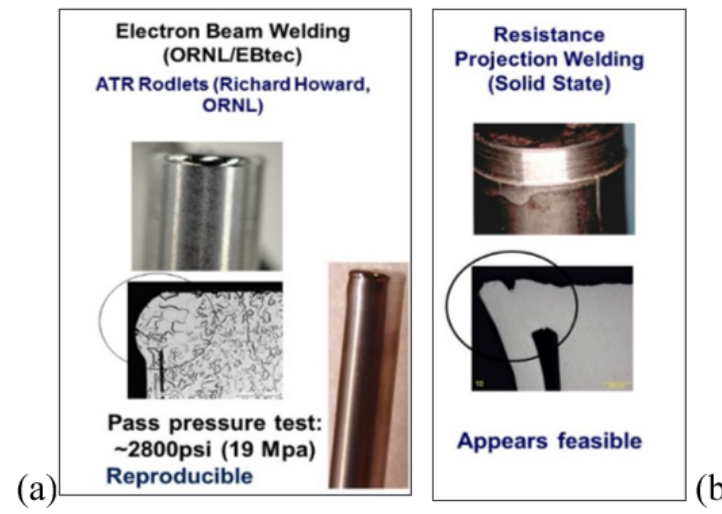

(b)

Fig. 6. Welded samples fabricated by (a) electron beam welding and (b) resistance projection welding.

fusion zone, as shown in Figure 6b. This technique is preferred for commercial deployment and will require further development.

\subsection{Corrosion resistance in simulated LWR coolants}

The corrosion resistance of pure Mo and Mo-ODS has been characterized in simulated BWR and PWR coolants at 288 and $330{ }^{\circ} \mathrm{C}$, respectively, in long-term autoclave tests $[8,9]$. Table 2 summarizes the corrosion data in LWR coolants. It is noted that the corrosion resistance of bare Mo-alloys in simulated BWR and PWR environments is excessively high and hence will require protection with a coating of Zr-alloy or $(\mathrm{Cr}, \mathrm{Al})$-containing stainless steel.

Preliminary results on Mo-alloys containing $\mathrm{Nb}$ have been found to significantly reduce the corrosion rate, as shown in Figure 7. Mo-alloy C containing 10\% Nb has an

Table 2. Summary of the corrosion rate (in $\mu \mathrm{m}$ per month) of bare and coated Mo-alloy tubes in simulated LWR coolants.

\begin{tabular}{llll}
\hline Test condition & Mo/ML & Zr-coated & FeCrAl-coated \\
\hline PWR $-330^{\circ} \mathrm{C}, 3.6 \mathrm{ppm} \mathrm{H} \mathrm{H}_{2}$ & $\sim 5$ & Very low & Very low \\
BWR-HWC $-288^{\circ} \mathrm{C}, 0.3 \mathrm{ppm} \mathrm{H} \mathrm{H}_{2}$ & $\sim 9$ & Very low & Very low \\
BWR-HWC $-288^{\circ} \mathrm{C}, 1 \mathrm{ppm} \mathrm{O} \mathrm{O}_{2}$ & $\sim 40$ & Very low & Very low \\
\hline
\end{tabular}




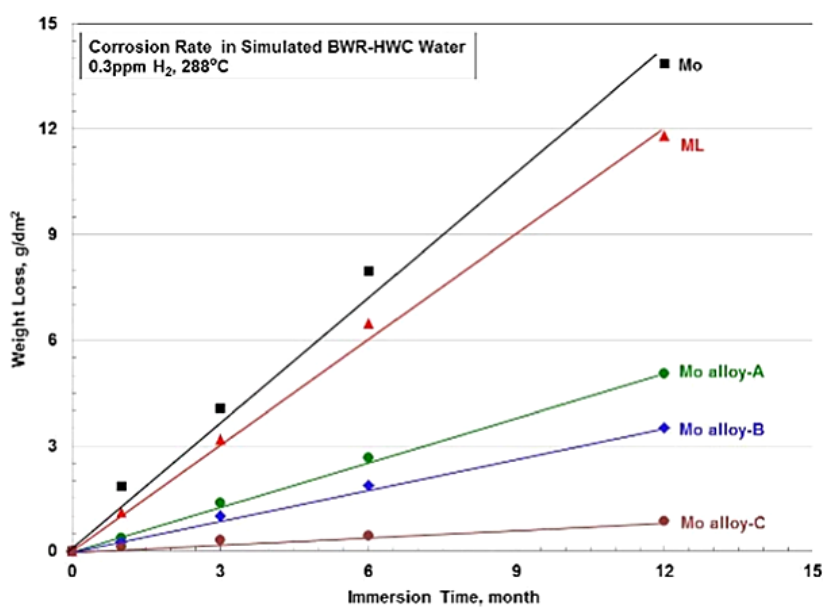

Fig. 7. Corrosion resistance of pure and ODS Mo and Nbcontaining Mo samples in simulated a BWR-HWC coolant.

order of magnitude lower corrosion rate $(\sim 0.5 \mu \mathrm{m} / \mathrm{mo})$ than that of the bare, pure Mo tube in a simulated BWR water containing $0.3 \mathrm{ppm}$ dissolved hydrogen.

\subsection{Oxidation resistance in $1000-1500{ }^{\circ} \mathrm{C}$ steam}

The oxidation resistance of uncoated Mo in oxidizing steam, i.e. steam containing free oxygen, has been known to be poor due to the formation of volatile $\mathrm{MoO}_{3}$ at temperature $>\sim 600^{\circ} \mathrm{C}$. In a severe loss of coolant accident, it is anticipated that the reactor core will have excess hydrogen due to corrosion and oxidation of various metallic components in the core. The $\mathrm{Zr}$-alloy and $\mathrm{FeCrAl}$ coatings are to provide protection from steam oxidation at elevated temperatures.

In pure steam and steam plus $10 \%$ hydrogen at $1000{ }^{\circ} \mathrm{C}$, uncoated Mo-alloy cladding has been found to have reasonable oxidation rate with a thicken loss of $\sim 20-25 \mu \mathrm{m}$ per day, which is two order of magnitude lower than that of $\mathrm{Zr}$ alloys $[9,10]$.

Coated Mo tubes with both-end welded received full protection from $\mathrm{FeCrAl}$ in $1000{ }^{\circ} \mathrm{C}$ for 7 days, shown in Figure 8. The Zircaloy- 4 coated Mo tube showed delamination of the $\mathrm{ZrO}_{2}$ and suffered some loss in the Mo wall thickness, but the Mo tube remains intact after 7 days.

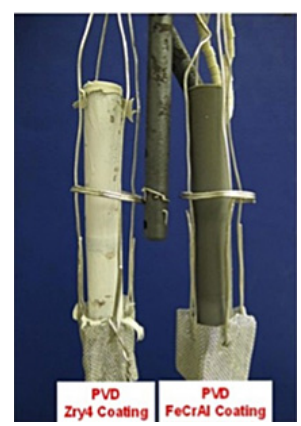

Fig. 8. Mo tubes with both-ends welded to endcaps and coated with either $\mathrm{FeCrAl}$ or Zircaloy-4 after testing in $1000^{\circ} \mathrm{C}$ steam for 7 days.

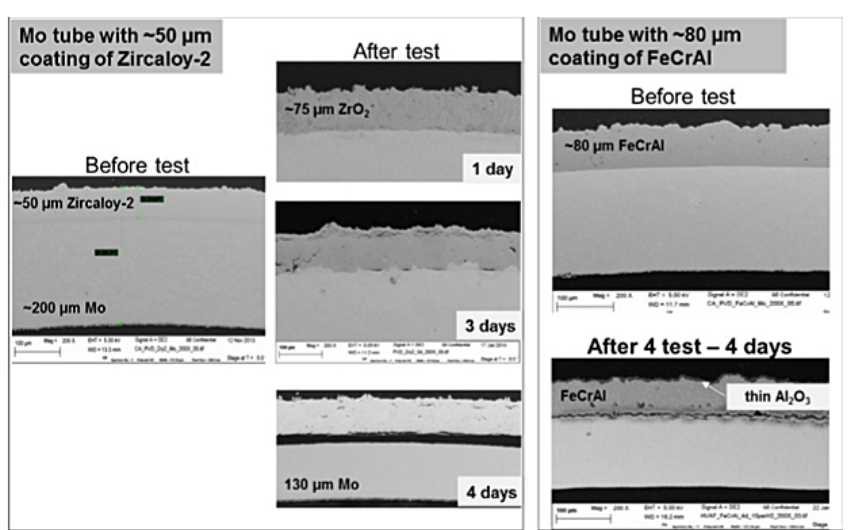

Fig. 9. Open-ended Mo-alloy tube samples with coating after oxidation test in $1000^{\circ} \mathrm{C}$ for up to 4 days.

Figure 8 shows cross-sections of open-ended Mo tube samples coated with either Zircaloy-2 or FeCrAl after being tested in $1000{ }^{\circ} \mathrm{C}$ steam for up to 4 days. The Zircaloy- 2 coated Mo tube shows the conversion of Zircaloy-2 to a dense $\mathrm{ZrO}_{2}$ which protects the Mo tube from steam oxidation for up to 4 days. The good integrity of the oxide formed from Zircaloy-2 differs from the flaky oxide formed from Zircaloy-4 as shown in Figure 9.

Investigation of the integrity of the $\mathrm{ZrO}_{2}$ oxide formed from the Zircaloy- 4 coating in Figure 8 and Zircaloy- 2 coating in Figure 9 has found that slightly over half of the alloying elements, Fe, Cr, Sn and Ni (Zircaloy-2 only), added to zirconium for corrosion and oxidation resistance were substantially lost during the CA-PVD coating process. This likely explains the flaky oxide observed in Figure 8. New target materials with substantially higher alloying concentrations have been obtained to improve the alloy chemistry of the coating. For the longer term, mechanical co-reduction should avoid the difficulty of controlling the alloy chemistry.

An interesting observation of the $1000{ }^{\circ} \mathrm{C}$ steam tested samples is the formation of an inter-diffusion zone in the FeCrAl-coated samples. The thickness was measured to be 2,4 , and $5 \mu \mathrm{m}$ after 1,3 , and 7 days, respectively. Clearly, the inter-diffusion was not significant enough to impact the cladding integrity at $1000^{\circ} \mathrm{C}$ for 7 days or much longer.

Tests in steam at $1200-1500^{\circ} \mathrm{C}$ has been in process.

\subsection{Irradiation properties}

Previous irradiation tests have shown Mo and its alloys, like other metals and alloys, will suffer from irradiation embrittlement and the mechanism has been attributed to grain boundary weakness. It is anticipated that improving the diametral mechanical properties through process and microstructure optimization, as discussed above, may lead to better properties after irradiation. Furthermore, additions of minor concentrations of alloying elements, such as $\mathrm{B}, \mathrm{Al}, \mathrm{Ti}$, and $\mathrm{Zr}$ have been suggested to strengthen the grain boundaries. New dilute Mo-alloys are being fabricated for testing.

Short rodlets with Mo and Mo-ODS cladding and 4\% enriched fuel pellets have been under preparation for 
irradiation in the ATR beginning in 2016. Additional irradiation in the Halden Reactor has been under preparation to begin in 2016 .

\section{Summary on results to date and challenges ahead}

This feasibility study of coated Mo-alloy cladding for accident tolerant fuel has demonstrated excellent corrosion in simulated LWR coolants and great oxidation properties in $1000^{\circ} \mathrm{C}$. The thin wall Mo tube has adequate mechanical strength and ductility, and further improvement in the diametral ductility is continuing to provide better resistance to pellet-to-cladding mechanical interaction. Fabrication of rodlets for irradiation is underway.

For commercial deployment, it may be necessary to fabricate the coated tubes via mechanical co-reduction, rather than relying on the PVD process, as the latter can be prohibitively expensive and challenging for quality control. A feasibility study on forming metallurgical bonding between the outer coating and the Mo-alloy via hot hydrostatic pressuring (Hipping), and subsequent mechanical co-reduction to produce coated Mo tubes has been underway. It is also necessary to incorporate fabrication process control to achieve optimized mechanical properties.

While the corrosion resistance of the coated cladding may be adequate, it is desirable to improve the corrosion resistance of the Mo tube to ensure full reliability of the cladding in the event of loss of the outer coating. To achieve this feature, Mo-alloys, primarily Nb-containing ones, are being studied.

The authors gratefully acknowledge the important contributions of Todd Leonhardt (Rhenium Alloys Inc.) for fabrication of thin wall Mo-alloy tubes; of Stu Malloy and Andy Nelson (LANL) for steam test studies; of Sam Armijo and Peter Ring for evaluation of induction heat treatment and hipping and metal bonding; of Kristine Barret of INL and Richard Howard of ORNL for supporting ATR irradiation of Mo cladded rodlets. The authors thank Jeff Deshon (EPRI) for management support. EPRI Fuel Reliability Program and EPRI Technology Innovation Program provide funding support. Areva has entered collaboration with EPRI on future Mo-alloy cladding development beginning in 2015. Generous collaborations from the US Department of Energy and the national laboratories are also appreciated.

\section{References}

1. B. Cheng, P. Chou, Y.-J. Kim, Enhancing fuel resistance to severe loss of coolant accidents with molybdenum-alloy fuel cladding, Paper 100075, in WRFPM2014, Sendai, Japan, September, 2014 (2014)

2. S. Bragg Sitten, Application of MELCOR to ATF concepts for severe accident analysis, in EPRI/INL/DOE workshop on Accident Tolerant Fuel, San Antonio, February, 2014 (2014)

3. B. Cheng, Fuel behavior in severe accidents and Mo-alloy based cladding to improve accident tolerance, Paper A0034, in TopFuel 2012, Birmingham, UK (2012)

4. IAEA Symposium on "Accident Tolerant Fuel Concepts For Light Water Reactors", Oak Ridge National Laboratory, Tennessee, October 13-16, 2014 (Proceedings to be published by IAEA)

5. B.V. Cockeram, R.W. Smith, K.J. Leonard, T.S. Byun, L.L. Snead, J. Nucl. Mater. 382, 1 (2008)

6. T.S. Byun, M. Li, B.V. Cockeram, L. Snead, J. Nucl. Mater. 376, 240 (2008)

7. G. Liu et al., Nanostructured high-strength molybdenum alloys with unprecedented tensile ductility, Nat. Mater. 12, $344(2013)$

8. Y.-J. Kim, B. Cheng, P. Chou, Molybdenum alloys for accident tolerant fuel cladding: high temperature corrosion and oxidation behavior, Paper 100144, in TopFuel 2014, September 14-17, 2014, Sendai, Japan (2014)

9. A.T. Nelson, E.S. Sooby, Y.-J. Kim, B. Cheng, S.A. Maloy, High temperature oxidation of molybdenum in water vapor environments, J. Nucl. Mater. 448, 441 (2013)

10. Y.-J. Kim, B. Cheng, P. Chou, Steam oxidation behavior of protective coatings on LWR Mo cladding for enhancing accident tolerance at high temperatures, Paper A0172, in TopFuel 2015, September 13-17, Zurich, Switzerland (2015)

Cite this article as: Bo Cheng, Peter Chou, Young-Jin Kim, Evaluations of Mo-alloy for light water reactor fuel cladding to enhance accident tolerance, EPJ Nuclear Sci. Technol. 2, 5 (2016) 